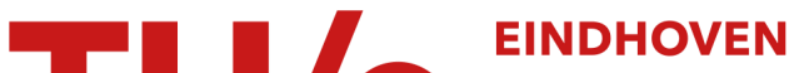 UNIVERSITY OF TECHNOLOGY
}

\section{Theory for spin diffusion in disordered organic semiconductors}

\section{Citation for published version (APA):}

Bobbert, P. A., Wagemans, W., Oost, van, F. W. A., Koopmans, B., \& Wohlgenannt, M. (2009). Theory for spin diffusion in disordered organic semiconductors. Physical Review Letters, 102(15), 156604-1/4. [156604]. https://doi.org/10.1103/PhysRevLett.102.156604

DOI:

10.1103/PhysRevLett.102.156604

Document status and date:

Published: 01/01/2009

\section{Document Version:}

Publisher's PDF, also known as Version of Record (includes final page, issue and volume numbers)

\section{Please check the document version of this publication:}

- A submitted manuscript is the version of the article upon submission and before peer-review. There can be important differences between the submitted version and the official published version of record. People interested in the research are advised to contact the author for the final version of the publication, or visit the $\mathrm{DOI}$ to the publisher's website.

- The final author version and the galley proof are versions of the publication after peer review.

- The final published version features the final layout of the paper including the volume, issue and page numbers.

Link to publication

\section{General rights}

Copyright and moral rights for the publications made accessible in the public portal are retained by the authors and/or other copyright owners and it is a condition of accessing publications that users recognise and abide by the legal requirements associated with these rights.

- Users may download and print one copy of any publication from the public portal for the purpose of private study or research.

- You may not further distribute the material or use it for any profit-making activity or commercial gain

- You may freely distribute the URL identifying the publication in the public portal.

If the publication is distributed under the terms of Article 25fa of the Dutch Copyright Act, indicated by the "Taverne" license above, please follow below link for the End User Agreement:

www.tue.nl/taverne

Take down policy

If you believe that this document breaches copyright please contact us at:

openaccess@tue.nl

providing details and we will investigate your claim. 


\title{
Theory for Spin Diffusion in Disordered Organic Semiconductors
}

\author{
P. A. Bobbert, ${ }^{1}$ W. Wagemans, ${ }^{1}$ F. W. A. van Oost,${ }^{1}$ B. Koopmans, ${ }^{1}$ and M. Wohlgenannt ${ }^{2}, *$ \\ ${ }^{1}$ Department of Applied Physics, Technische Universiteit Eindhoven, P.O. Box 513, 5600 MB Eindhoven, The Netherlands \\ ${ }^{2}$ Department of Physics and Astronomy and Optical Science and Technology Center, University of Iowa, \\ Iowa City, Iowa 52242-1479, USA
}

(Received 2 January 2009; published 17 April 2009)

\begin{abstract}
We present a theory for spin diffusion in disordered organic semiconductors, based on incoherent hopping of a charge carrier and coherent precession of its spin in an effective magnetic field, composed of the random hyperfine field of hydrogen nuclei and an applied magnetic field. From Monte Carlo simulations and an analysis of the waiting-time distribution of the carrier we predict a surprisingly weak temperature dependence, but a considerable magnetic-field dependence of the spin-diffusion length. We show that both predictions are in agreement with experiments on organic spin valves.
\end{abstract}

PACS numbers: 72.25.Dc, 72.25.Rb

The study of electron-spin transport through nonmagnetic spacer materials in between ferromagnetic electrodes is an extremely active field, because of the rich physics involved and the important applications in the area of magnetic sensors [1]. If the spin-diffusion length is larger than or comparable to the distance between the electrodes, the current through such structures depends strongly on the mutual orientation of the magnetizations of the electrodes, which is called the spin-valve effect, leading to "giant magnetoresistance" (GMR) [2,3].

Traditionally, nonmagnetic metals are used as the spacer-layer material in these structures. Spintronic devices utilizing spin injection and transport through a semiconducting spacer layer offer additional functionalities, such as spin transistors and the possibility to realize quantum computation logic. Consequently, much effort is put into finding suitable materials. Spin relaxation in the inorganic materials traditionally used in these structures, containing relatively heavy atoms, is mainly caused by spin-orbit coupling [4]. Organic semiconductors (OS) are a very interesting alternative because of the enormous versatility of organic chemistry and because the light atoms from which they are composed cause very little spin-orbit coupling [5]. Recent years have seen the first demonstrations of GMR devices [6-12] as well as magnetic tunnel junctions [13] using OS as spacer layer. Here we will investigate the former type of devices. The experiments reported so far have shown that when the thickness of the OS spacer layer increases, the GMR effect in these devices disappears on a typical length scale of the order of 10$100 \mathrm{~nm}$. Two very important and still unanswered questions, addressed in this Letter, are what is the cause of the remaining spin relaxation and what factors determine the spin-diffusion length?

Recent research on magnetic-field effects on the resistance and luminescence of OS has led to the conclusion that the hydrogen hyperfine fields are involved, influencing reactions between spin-carrying radicals (polarons, triplet excitons) [14-17]. The accurate prediction of magnetore- sistance line shapes assuming coupling of the spin to these hyperfine fields [17] strongly suggests that this coupling is the main source of spin relaxation in OS. In this Letter, we take this as our working hypothesis. Since typically many ( $\sim 10$ or more) hydrogen nuclear spins couple to the spin of a charge carrier in OS, we can replace the hyperfine coupling by a classical, quasistatic, and random field, distributed according to a three-dimensional Gaussian [18], with standard deviation $B_{\mathrm{hf}}$. In addition, we model charge transport in disordered OS by hopping of carriers between localized sites with random site energies, distributed according to a Gaussian density of states (GDOS) with standard deviation $\sigma$ [19]. Hence, we describe spin diffusion in these materials by a combination of incoherent hopping of a carrier in a GDOS together with coherent precession of its spin $\mathbf{S}(t)$ around a local effective magnetic field; see Fig. 1(a). At each hopping site $i$ this effective field is $\mathbf{B}_{i}=$ $\mathbf{B}_{\mathrm{hf}, i}+\mathbf{B}$, where $\mathbf{B}_{\mathrm{hf}, i}$ is the random hyperfine field at this site and $\mathbf{B}=B \hat{\mathbf{z}}$ the externally applied magnetic field, e.g., the field to which a GMR sensor should respond. With typically $B_{\mathrm{hf}} \approx 5 \mathrm{mT}$ the hyperfine precession frequency is $\omega_{\mathrm{hf}}=\gamma B_{\mathrm{hf}} \approx 10^{8} \mathrm{~s}^{-1}$ ( $\gamma$ is the gyromagnetic ratio).

We consider the situation that a carrier with unit charge $e$ (electron or hole) and spin fully polarized in the $z$ direction is injected by an electrode into the organic material at $x=$ 0 and moves to the opposite electrode under the influence of an electric field $\mathbf{E}=E \hat{\mathbf{x}}$. We assume that nearestneighbor hopping takes place by thermally assisted tunneling [20] from site $i$ to $j$ with a rate $\omega_{i j}=\omega_{\text {hop }} \exp \left[-\left(\varepsilon_{j}-\right.\right.$ $\left.\left.\varepsilon_{i}\right) / k_{B} T\right]$ for $\varepsilon_{j} \geq \varepsilon_{i}$ and $\omega_{i j}=\omega_{\text {hop }}$ for $\varepsilon_{j}<\varepsilon_{i}$, where $T$ is temperature, $k_{B}$ is Boltzmann's constant, and $\varepsilon_{i}$ and $\varepsilon_{j}$ are the on-site-energies of sites $i$ and $j$, with a contribution due to the electric field added. The prefactor $\omega_{\text {hop }}$ contains a phonon attempt frequency as well as a factor related to a wave function overlap. For hopping in disordered OS at not too low temperatures it is a good approximation to include only nearest-neighbor hopping [21]. Furthermore, it has been shown that positional disorder is much less important than energetic disorder [19], so for simplicity we neglect 


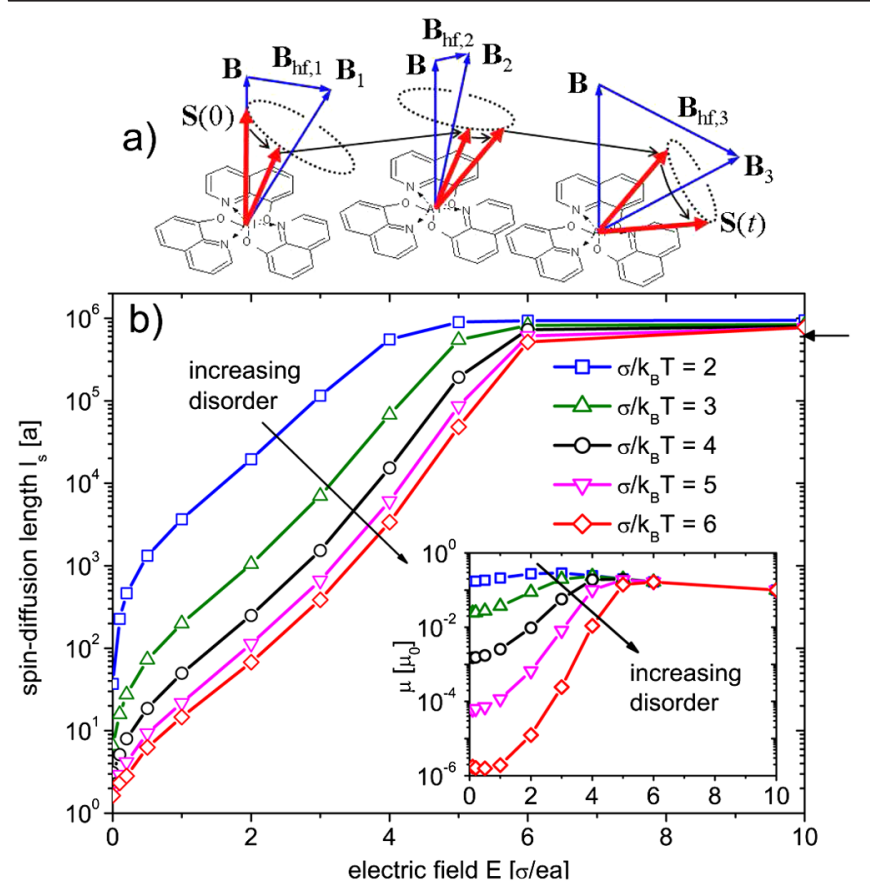

FIG. 1 (color online). (a) Mechanism for spin diffusion, in this case for the molecular semiconductor $\mathrm{Alq}_{3}$. (b) Spin-diffusion length $l_{s}$ [as multiples of the lattice constant] vs electric field $E$ at zero magnetic field, for $r \equiv \omega_{\text {hop }} / \omega_{\text {hf }}=1000$ and different $\hat{\sigma} \equiv \sigma / k_{B} T$. The arrow at the right axis indicates the result for a one-dimensional chain at large $E$. Inset: corresponding mobility $\mu$ vs $E$, in units of $\mu_{0} \equiv e a^{2} \omega_{\text {hop }} / \sigma$.

positional disorder and take a fixed nearest-neighbor distance $a$.

Clearly, the ratio $r \equiv \omega_{\text {hop }} / \omega_{\text {hf }}$ is an important parameter. If $r$ is large the influence of the hyperfine field is small and large spin-diffusion lengths can be expected, while the opposite holds if $r$ is small. For derivatives of the familiar $\pi$-conjugated polymer poly-(para-phenylene vinylene) (PPV) we obtain an estimate of $10^{9}-10^{11} \mathrm{~s}^{-1}$ for $\omega_{\text {hop }}$ [21]. Hence, for this class of organic semiconductors $r$ is of the order of 10-1000, but the large variation even within this class shows that very different values can be expected for different organic semiconductors.

It is instructive to first consider a one-dimensional (1D) chain of sites for the case $E \gg \sigma / e a$, when all hops are down-field with the same rate $\omega_{\text {hop }}$, leading to equal average waiting times $1 / \omega_{\text {hop }}$ at each site. By solving the timedependent Schrödinger equation for the spinor in the effective magnetic field and performing an average over the random hyperfine fields (in cylindrical coordinates $\rho, \zeta$ ) [22], one can easily derive the following expression for the relative preservation, $\lambda$, of spin polarization, $p$, during the waiting time of the carrier at a site:

$$
\begin{aligned}
\lambda & =\frac{1}{\sqrt{2 \pi}} \int_{0}^{\infty} \rho d \rho \int_{-\infty}^{\infty} d \zeta e^{-\left(\rho^{2}+\zeta^{2}\right) / 2} \frac{r^{2}+(\zeta+b)^{2}}{r^{2}+\rho^{2}+(\zeta+b)^{2}} \\
& \approx \exp \left\{-\left[1 / \ln (3)+r^{2} / 2+b^{2} / 2\right]^{-1}\right\}
\end{aligned}
$$

with $b \equiv B / B_{\mathrm{hf}}$. This leads to an exponentially decaying polarization $p(x)=\exp \left(-x / l_{s}\right)$, with a spin-diffusion length $l_{s}=-a / \ln \lambda \approx a\left[1 / \ln (3)+r^{2} / 2+b^{2} / 2\right]$. The increase of $l_{s}$ with increasing $b$ and $r$ can readily be understood qualitatively: with increasing $b$, the Zeeman coupling becomes increasingly dominant over the hyperfine coupling and the carrier spin becomes effectively pinned. The quadratic increase with $r$ results from "motional narrowing", well-known in magnetic-resonance spectroscopy.

For the three-dimensional (3D) situation we performed Monte Carlo simulations for hopping of a single carrier in a homogeneous electric field of arbitrary magnitude on a cubic lattice of sites $(N \times 50 \times 50$, where $N$ is adapted to the specific situation), while simultaneously solving the time-dependent Schrödinger equation for its spinor [22]. The random site-energies and hyperfine fields are drawn from their corresponding Gaussian distributions. Sufficiently far from the injecting layer, $p(x)$ decreases exponentially, from which we extract $l_{s}$. We averaged over several thousands of energetic and hyperfine disorder configurations, making sure that the error bar in the plots discussed below is smaller than the symbol sizes shown.

In Fig. 1(b) we show $l_{s}$ as a function of $E$ for different reduced disorder strengths $\hat{\sigma} \equiv \sigma / k_{B} T$, for $r=1000$ and $B=0$. The arrow at the right axis shows the value obtained for the 1D model discussed above, which is quite close to the present results at large $E$. For other values of $r \gg 1$ (fast hopping) we find a similar dependence on $E$ as shown in Fig. 1(b). For $r \leq 1$ (slow hopping) we find $l_{s} \sim a$, indicating that for this case no significant spin-diffusion length is found. The relevant case for the experiments carried out up to now [6-12] corresponds to $E \ll \sigma / e a$, so from now on we will focus on this case. We note that whereas $l_{s}$ decreases steeply for $E \rightarrow 0$, it remains nonzero at $E=0$.

The inset in Fig. 1(b) shows the carrier mobility $\mu$ as a function of $E$ (for comparison: the unit $\mu_{0}$ is of the order of $10^{-7}-10^{-5} \mathrm{~m}^{2} / \mathrm{V} \mathrm{s}$ for the PPV derivatives studied in Ref. [21]). A strikingly different trend with increasing $\hat{\sigma}$ is observed for $l_{s}$ compared to $\mu$ at small and intermediate $E$ : while $\mu$ keeps on decreasing very rapidly with increasing $\hat{\sigma}, l_{s}$ depends rather weakly on $\hat{\sigma}$ and even appears to saturate for large $\hat{\sigma}$. In order to provide an explanation for the weak dependence of $l_{s}$ on $\hat{\sigma}$ we show in Fig. 2(a) the distribution $P(\tau)$ of waiting times $\tau$ [23] of the carrier while hopping through the lattice, at $E=0$ and different $\hat{\sigma}$. The curves appear to saturate for large $\hat{\sigma}$. By considering hops upwards in energy from a Boltzmann distribution in the GDOS one can in fact prove [24] that $P(\tau)$ converges for large $\tau$ to a universal algebraic distribution $P(\tau) \sim$ $\tau^{-3 / 2}$ in the limit $\hat{\sigma} \rightarrow \infty$, see the dashed line in Fig. 2(a). We note that $P(\tau)$ for a GDOS has been studied before [25], but for too small disorder strengths $(\hat{\sigma} \leq 4)$ to observe this universal behavior.

The quite different dependencies of $l_{s}$ and $\mu$ on $\hat{\sigma}$ can now be understood as follows. With increasing $\hat{\sigma}$ the tail of 

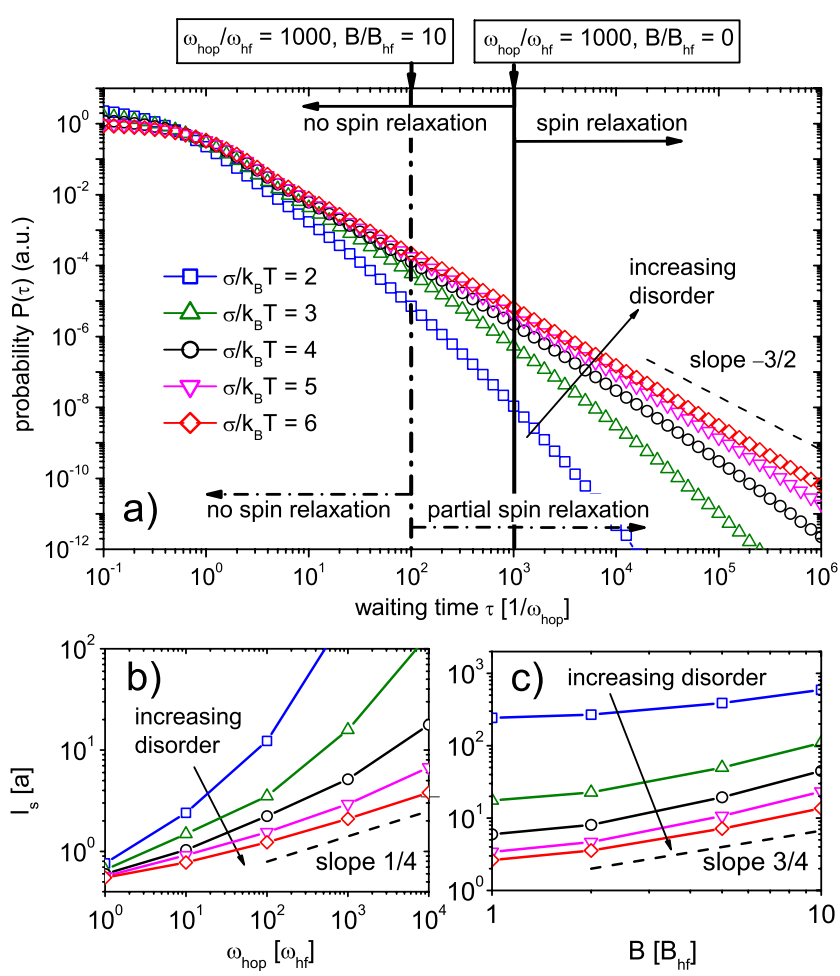

FIG. 2 (color online). (a) Waiting-time distributions (equally normalized) at $E=0$ for different $\hat{\sigma} \equiv \sigma / k_{B} T$. Solid and dashdotted lines and arrows: see the main text. (b) $l_{s}$ at $E=0.1 \sigma / e a$ as a function of $\omega_{\text {hop }}$ at $B=0$ and (c) as a function of magnetic field $B$ for $r \equiv \omega_{\text {hop }} / \omega_{\text {hf }}=1000$. The dashed lines in (a)-(c) indicate the expected power laws.

the distribution $P(\tau)$ contains an increasing amount of sites with very large $\tau$, leading to a strong decrease of the mobility, since these sites cause a very long delay in the motion of the carrier. Regarding the spin diffusion, however, the situation is distinctly different. Let us, for example, consider the case $r=1000$ and $B=0$. At sites $i$ with $\tau_{i}$ to the left of the solid line in Fig. 2(a), such that $r_{i} \equiv 1 / \omega_{\mathrm{hf}} \tau_{i} \gg 1$, essentially no polarization loss occurs [cf. Eq. (1) with $r$ replaced by $r_{i}$ ]. At sites with $\tau_{i}$ to the right of this line, such that $r_{i} \ll 1$, almost immediate polarization loss occurs, but this effect is essentially the same for all these sites. For large $\hat{\sigma}$ the fraction of the latter sites, obtained by integrating $P(\tau)$ from the solid line to the right, converges to $\sim 1 / r^{1 / 2}$. This means that on average the spin polarization disappears in $\sim r^{1 / 2}$ hops. Since at small $E$ diffusion of carriers is dominant over drift one expects $l_{s} \sim\left(r^{1 / 2}\right)^{1 / 2} \sim r^{1 / 4}$. The dashed line in Fig. 2(b) indicates this expected power law, to which the results indeed converge (for numerical reasons we took a small but finite $E$ ).

In Fig. 2(c) we plot $l_{s}$ at small $E$ as a function of magnetic field $B$, for $r=1000$. For $B>B_{\mathrm{hf}}$ we observe an important $B$-dependence, which again converges to a power law for increasing $\hat{\sigma}$. The analysis now goes as follows. Let us, for example, take $b=B / B_{\mathrm{hf}}=10$. From Eq. (1) (again replacing $r$ by $r_{i} \equiv 1 / \omega_{\mathrm{hf}} \tau_{i}$ ) it follows that if $r_{i} \gg b$, i.e., to the left of the dash-dotted line in Fig. 2(a), basically no polarization loss takes place. To the right of this line partial polarization loss takes place with $1-\lambda \sim 1 / b^{2}$, which is a consequence of the pinning effect of the Zeeman term discussed above. A similar argument as above now leads to the expectation $l_{s} \sim b^{3 / 4}$ in the diffusive regime, which is seen to be very well obeyed. Together with the above power-law dependence on $r$ this allows us to estimate $l_{s}$ for general values of $r \gg 1$ and $b$ for the case of large $\hat{\sigma}$ and small $E$.

We now undertake a comparison between our theory and experimental results, as far as they are available at the moment. We have no information about the hopping frequencies of the OS used in the spin valves of Refs. [6-8]: sexithienyl, tris-(8-hydroxyquinoline) aluminum $\left(\mathrm{Alq}_{3}\right)$, and poly-3-hexylthiophene, respectively. Since the mobilities in these materials are higher than those of the PPVderivatives investigated in Ref. [21], we expect that the hopping frequencies are such that $r>1000$. A calculated value of $\sigma=0.35 \mathrm{eV}$ for the energetic disorder of electrons in $\mathrm{Alq}_{3}$ [26] leads to $\hat{\sigma} \approx 14$ at room temperature, which is clearly in the strong-disorder limit. We can conclude that with a typical value $a \approx 1 \mathrm{~nm}$ the spin-diffusion lengths of about 10-100 $\mathrm{nm}$ found in Refs. [6-8] and recently confirmed with muon spin-resonance studies [10] are compatible with our results.

Very interestingly, inspection of the experimental GMR traces in Refs. [7,8], both using $\mathrm{La}_{0.67} \mathrm{Sr}_{0.33} \mathrm{MnO}_{3}$ (LSMO) and $\mathrm{Co}$ as electrodes, reveals that in the up- and down-field sweeps the resistance as a function of $B$ changes considerably already before the magnetization of the soft layer (with the weaker coercive field) is reversed. This points at a source of magnetoresistance other than the switching of the ferromagnetic layers and we propose that this is the magnetic-field dependence of the spin-diffusion length predicted by our theory. To illustrate its effect on the GMR traces, we simulated such traces using a simple phenomenological model. We describe the organic film by a reservoir for carriers with spin up and down, link the finite spin-diffusion length to the spin-flip rate between the two reservoirs, and derive an equation for the spin accumulation by solving the rate equations [22]. We fitted the resulting GMR traces to experimental data of Ref. [7]; see Fig. 3. The specific equation used in the fit reads: $\operatorname{MR}(B)=\operatorname{MR}_{\max } \frac{1}{2}\left(1+m_{1}(B) m_{2}(B)\right) \exp \left[-d / l_{s}(B)\right]$,

with $\mathrm{MR}_{\max }$ the MR when neglecting spin relaxation, $d$ the thickness of the organic spacer, and we explicitly accounted for the fact that the system studied displays an opposite spin polarization for the parallel alignment of the magnetizations. The magnetization of electrode $i=1,2$ is represented by $m_{i}(B)$, measured along the applied field direction and normalized to the saturation magnetization. In this case we used an error function centered at the coercive field to match the rounded switching of the respective electrodes. For the $B$ dependence of $l_{s}$ we assumed $l_{s}(B)=l_{s, 0}\left[\sqrt{1+\left(B / B_{0}\right)^{2}}\right]^{3 / 4}$ with $B_{0}=2.3 B_{\mathrm{hf}}$, 


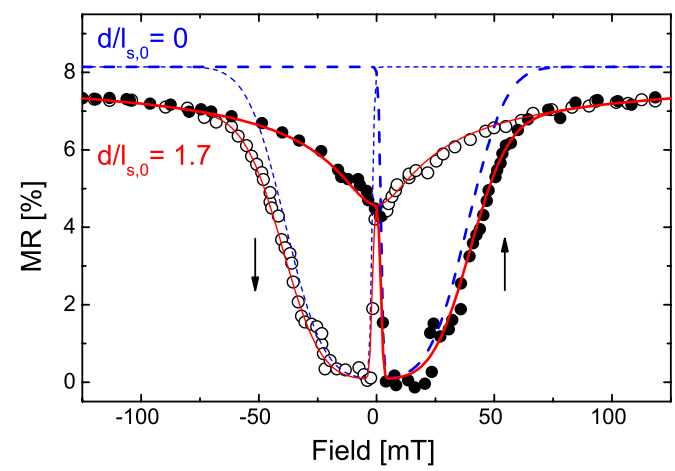

FIG. 3 (color online). Full curves: fit of the model discussed in the main text to the experimental magnetoresistance (MR) traces taken from Fig. 3b of Ref. [7] (symbols). Dashed curves: results neglecting spin relaxation. Thick/thin lines: up-/down-field sweep.

as predicted by our theory in the diffusive regime $(E \ll$ $\sigma / e a$, see Fig. 2(c) and the fit in Ref. [22]). Figure 3 shows that this predicted $l_{s}(B)$ accurately reproduces the shape of $\operatorname{MR}(B)$ in the region before switching of the soft layer, with a minimum number of parameters. The fitting procedure yields $l_{s, 0} \approx 1.7 d$ and $B_{\mathrm{hf}} \approx 5.7 \mathrm{mT}$ [22], the latter being indeed a typical value for the random hyperfine field. The simulated MR-trace for $d / l_{s, 0}=0$ (no spin relaxation) deviates strongly from the experimental data, demonstrating that the latter cannot be explained assuming a $B$-independent spin-diffusion length together with an injected spin polarization proportional to the reported electrode magnetization.

Our theory predicts a rather weak dependence of $l_{s}$ on the relative disorder strength $\sigma / k_{B} T$ and hence on temperature, in agreement with experiments $[7,11,12]$. In the experiments with LSMO as one of the electrodes the GMR effect decreases significantly above $T \approx 100 \mathrm{~K}$, but this can be fully attributed to a reduction of the spin polarization of the injected current $[11,12]$. Finally, it is important to note that in the experiments the GMR effect rapidly disappears with growing bias voltage $[7,11,12]$ on a voltage scale $(\sim 1 \mathrm{~V})$ that corresponds in our theory to electric fields for which $e E a / \sigma \ll 1$. At such fields, $l_{s}$ has saturated to its value at $E=0$. Therefore, our view is that the measured bias-voltage dependence is not caused by a dependence of $l_{s}$ on the electric field. Several possible explanations for the steep decrease of spin-valve efficiency with increasing bias have been suggested [11], but it is clear that this issue requires much further study. If efficient spin injection can be realized with high biases such that $e E a / \sigma>1$, our theory predicts greatly enhanced spindiffusion lengths of the order of several hundreds of nanometers, up to even millimeters [see Fig. 1(a)].

In summary, we have presented a theory for spin diffusion in disordered organic semiconductors with hyperfine coupling, based on a combination of incoherent carrier hopping and coherent spin precession in a random effective magnetic field. We obtain spin-diffusion lengths of the correct magnitude that depend rather weakly on temperature, but considerably on the applied magnetic field, in agreement with experiments on spin valves.

We thank Professor Heinz Bässler for a helpful discussion about the waiting-time distribution. This work was supported by the Dutch Technology Foundation (STW) via the NWO VICI-Grant "Spin Engineering in Molecular Devices", by NSF Grant No. ECS 07-25280 and by Army MURI Grant No. W911NF-08-1-0317.

*markus-wohlgenannt@uiowa.edu

[1] S. A. Wolf et al., Science 294, 1488 (2001).

[2] M. N. Baibich et al., Phys. Rev. Lett. 61, 2472 (1988).

[3] G. Binasch, P. Grünberg, F. Saurenbach, and W. Zinn, Phys. Rev. B 39, 4828 (1989).

[4] G. Pikus and A. Titkov, Optical Orientation, edited by F. Meier and B.P. Zakharchenya (North-Holland, Amsterdam, 1984), Chap. 3.

[5] A. R. Rocha et al., Nature Mater. 4, 335 (2005).

[6] V. Dediu et al., Solid State Commun. 122, 181 (2002).

[7] Z. H. Xiong, D. Wu, Z. V. Vardeny, and J. Shi, Nature (London) 427, 821 (2004).

[8] S. Majumdar et al., Appl. Phys. Lett. 89, 122114 (2006).

[9] J. R. Petta, S. K. Slater, and D. C. Ralph, Phys. Rev. Lett. 93, 136601 (2004).

[10] A. J. Drew et al., Nature Mater. 8, 109 (2009).

[11] F. J. Wang et al., Phys. Rev. B 75, 245324 (2007).

[12] V. Dediu et al., Phys. Rev. B 78, 115203 (2008).

[13] T. S. Santos, J. S. Lee, P. Migdal, I. C. Lekshmi, B. Satpati, and J. S. Moodera, Phys. Rev. Lett. 98, 016601 (2007).

[14] J. Kalinowski et al., Chem. Phys. Lett. 380, 710 (2003).

[15] V. Prigodin, J. Bergeson, D. Lincoln, and A. Epstein, Synth. Met. 156, 757 (2006).

[16] P. Desai et al., Phys. Rev. B 75, 094423 (2007).

[17] P. A. Bobbert, T. D. Nguyen et al., Phys. Rev. Lett. 99, 216801 (2007).

[18] K. Schulten and P. Wolynes, J. Chem. Phys. 68, 3292 (1978).

[19] H. Bässler, Phys. Status Solidi B 175, 15 (1993).

[20] A. Miller and E. Abrahams, Phys. Rev. 120, 745 (1960).

[21] W. F. Pasveer et al., Phys. Rev. Lett. 94, 206601 (2005).

[22] See EPAPS Document No. E-PRLTAO-102-054918 for supplementary material. For more information on EPAPS, see http://www.aip.org/pubservs/epaps.html.

[23] The concept of a waiting-time distribution was originally introduced in the field of dispersive transport; see H. Scher and E. W. Montroll, Phys. Rev. B 12, 2455 (1975).

[24] P. A. Bobbert, F. W. A. van Oost, and J. Cottaar (to be published).

[25] B. Hartenstein et al., Phys. Rev. B 54, 8574 (1996).

[26] Y. Nagata and C. Lennartz, J. Chem. Phys. 129, 034709 (2008). 